The Astrophysical Journal, 592:L45-L48, 2003 July 20

(c) 2003. The American Astronomical Society. All rights reserved. Printed in U.S.A.

\title{
CHANDRA DETECTION OF A PULSAR WIND NEBULA ASSOCIATED WITH SUPERNOVA REMNANT 3C 396
}

\author{
C. M. Olbert, ${ }^{1,2}$ J. W. Keohane, ${ }^{1,2,3}$ K. A. Arnaud,${ }^{4}$ K. K. Dyer, ${ }^{5}$ S. P. Reynolds, ${ }^{6}$ and S. Safi-Harb ${ }^{7}$ \\ Received 2002 May 31; accepted 2003 June 6; published 2003 June 26
}

\begin{abstract}
We present a 100 ks observation of the Galactic supernova remnant 3C 396 (G39.2-0.3) with the Chandra $X$-Ray Observatory that we compare with a $20 \mathrm{~cm}$ map of the remnant from the Very Large Array. In the Chandra images, a nonthermal nebula containing an embedded pointlike source is apparent near the center of the remnant that we interpret as a synchrotron pulsar wind nebula surrounding a yet undetected pulsar. From the $2-10 \mathrm{keV}$ spectrum for the nebula $\left[N_{\mathrm{H}}=(5.3 \pm 0.9) \times 10^{22} \mathrm{~cm}^{-2}, \Gamma=1.5 \pm 0.3\right]$ we derive an unabsorbed X-ray flux of $S_{\mathrm{x}}=1.62 \times 10^{-12} \mathrm{ergs} \mathrm{cm}^{-2} \mathrm{~s}^{-1}$, and from this we estimate the spin-down power of the neutron star to be $\dot{E}=7.2 \times 10^{36} \mathrm{ergs} \mathrm{s}^{-1}$. The central nebula is morphologically complex, showing bent, extended structure. The radio and X-ray shells of the remnant correlate poorly on large scales, particularly on the eastern half of the remnant, which appears very faint in X-ray images. At both radio and X-ray wavelengths, the western half of the remnant is substantially brighter than the eastern half.
\end{abstract}

Subject headings: ISM: individual (G39.2-0.3) — pulsars: general — stars: neutron — supernova remnants

\section{INTRODUCTION}

The origin of the central emission in the Galactic supernova remnant (SNR) 3C 396 (G39.2-0.3) has been under debate for two decades. Caswell et al. (1982) first classified it as a Crab-like SNR because of its bright central radio emission, despite the fact that its steep spectral index $\left(\alpha \sim 0.49 ; S_{\nu} \propto\right.$ $\nu^{-\alpha}$ ) was typical of shell-type SNRs. On the other hand, the Einstein X-ray data of Becker \& Helfand (1987) did not convincingly distinguish a nonthermal spectral component. In addition, their Very Large Array (VLA) ${ }^{8}$ radio data showed no radio spectral index variations across the source, and they concluded that the central emission was likely caused by a shell filament projected across the center of the SNR.

Patnaik et al. (1990) presented VLA polarization data that show, in addition to the clear shell morphology, a highly polarized region near the center of the SNR, but they did not consider that the emission might be powered by a pulsar. Patnaik et al. also made a determination of the distance to the source, agreeing with the distance estimate of $d=6.5-9.6 \mathrm{kpc}$ made by Caswell et al. (1975; corrected for a Galactocentric radius of $8.5 \mathrm{kpc}$ ). For convenience, we express distancedependent quantities in terms of $d_{8}$, the distance to 3C 396 in units of $8 \mathrm{kpc}$, our best estimate (i.e., $d_{8}=d / 8 \mathrm{kpc}$ ).

Anderson \& Rudnick (1993) presented VLA data of the spectral index between 6 and $20 \mathrm{~cm}$, using a novel sliding block regression method intended to mitigate the problems due to systematic errors between the two frequencies. They found a region of flat spectral index near the center of 3C 396 and discussed a number of possibilities for its origin, including that

\footnotetext{
${ }^{1}$ North Carolina School of Science and Mathematics, 1219 Broad Street, Durham, NC 27705.

${ }^{2}$ Department of Physics and Astronomy, University of North Carolina, Chapel Hill, NC 27599.

${ }^{3}$ Current Address: SIRTF Science Center, California Institute of Technology, MS 220-6, 1200 East California Boulevard, Pasedena, CA 91125.

${ }^{4}$ Goddard Space Flight Center, Code 662, Greenbelt, MD 20771.

${ }^{5}$ National Radio Astronomy Observatory, P.O. Box O, Soccoro, NM 87801

${ }^{6}$ North Carolina State University, Box 8202, Raleigh, NC 27695-8202.

${ }^{7}$ NSERC UFA fellow: University of Manitoba, Winnipeg, MB R3T 2N2, Canada.

${ }^{8}$ The VLA is operated by the National Radio Astronomy Observatory, a facility of the National Science Foundation operated under cooperative agreement by Associated Universities, Inc.
}

it is an underlying plerionic region. More recently, Harrus \& Slane (1999) distinguished a nonthermal component in the ASCA spectrum of 3C 396 (coincident with the spectrally flat region of Anderson \& Rudnick and the highly polarized region of Patnaik et al.) that they attributed to a synchrotron pulsar wind nebula $(\mathrm{PWN})$ powered by a rapidly rotating neutron star. If the neutron star emits in $\gamma$-rays as well, this interpretation could also explain the unidentified EGRET source (2EGS J1903 + 0529; Yadigaroglu \& Romani 1997), based on its value of $\dot{E} / 4 \pi d^{2} \sim 10^{-9}$ ergs $\mathrm{cm}^{-2} \mathrm{~s}^{-1}$, which is typical of X-ray pulsars and also reasonable for some $X$-ray $/ \gamma$-ray pulsars (Thompson 2000), although previous searches for pulsed emission have been unsuccessful (e.g., Biggs \& Lyne 1996; Gorham et al. 1996).

In $\S 2.1$, we present spectra and high-resolution images of 3C 396 obtained with the Chandra X-Ray Observatory and compare them with a $20 \mathrm{~cm}$ radio map from the VLA. In $\S 2.2$, we analyze the spectra of the central nebula independent of the shell spectrum (the discussion of which is deferred to another paper). We argue in $\S 3$ that the central nebula seen with Chandra is most tenably interpreted as a PWN associated with the SNR 3C 396.

\section{OBSERVATIONS AND ANALYSIS}

3C 396 was observed by the Chandra X-Ray Observatory for $100 \mathrm{ks}$ on 2001 March 12 with the seventh chip of the Advanced CCD Imaging Spectrometer array (ACIS-S3) at a focal plane temperature of $-120^{\circ} \mathrm{C}$. The data were corrected for charge transfer inefficiency with tools provided by the ACIS Instrument Team of Penn State University (Townsley et al. 2000). The gain-map calibration was reapplied according to standard CIAO version 2.2.1 processing procedures (using ver. 2.10 of the CALDB calibration files), and only those events with standard ASCA grades (02346) were retained. Brief periods of anomalous background rates were rejected, giving a total exposure time of $96 \mathrm{ks}$ after processing.

\subsection{Images}

3C 396 is resolved by Chandra as an elliptical, diffuse source with some limb brightening, approximately $6.5 \times 4^{\prime}$ in size, with a bright, compact region in the interior. In contrast, the 


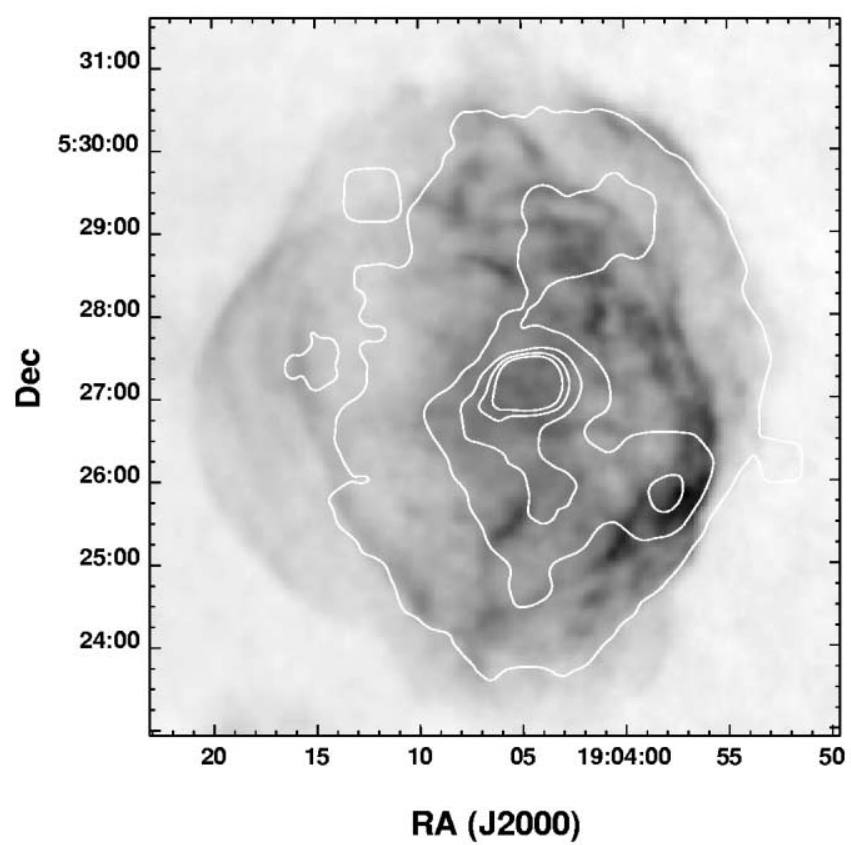

FIG. 1.-A $20 \mathrm{~cm}$ VLA (Dyer \& Reynolds 1999a) gray-scale image smoothed with a 6". $1 \times 6$ " .8 beam with broadband X-ray $(0.3-8.0 \mathrm{keV})$ contours (smoothed by 6."5 FWHM) overlaid. The contours are in five linearly decreasing steps from a maximum defined by the remnant emission away from the nebula. We defer a detailed discussion of the morphological comparison between the two wavelengths to a future paper and note only the lack of any corresponding feature at the location of the PWN in the radio image.

SNR is manifested in the radio band as a circular projected shell of $\sim 7^{\prime}$ diameter with centrally brightened emission as well as apparent limb brightening along the western edge. The eastern portion of the shell shows only very faint low surface brightness X-ray emission and is also slightly less bright in the radio. In Figure 1, we show a gray-scale image of the $20 \mathrm{~cm}$ radio map (Dyer \& Reynolds 1999a; see also Patnaik et al. 1990 and Anderson \& Rudnick 1993) overlaid with broadband X-ray contours $(0.3-8.0 \mathrm{keV})$. Figure 2 plots the broadband $\mathrm{X}$-ray emission scaled to emphasize the shell. As noted previously, discussion of the shell and shell spectrum is deferred by the authors to a future paper.

The central nebula visible in the X-ray, centered at coordinates (J2000) $\alpha=19^{\mathrm{h}} 04^{\mathrm{m}} 04^{\mathrm{s}} .7, \delta=05^{\circ} 27^{\prime} 12^{\prime \prime}$, is the most prominent feature in the Chandra images. The nebula extends approximately $55^{\prime \prime}$ in diameter along the east-west axis and approximately $20^{\prime \prime}$ in diameter north-south, corresponding to dimensions of $2.2 \times 0.8 d_{8} \mathrm{pc}$. A pointlike source is detected at the center of the nebula at the coordinates cited above, and there are three extensions away from the pointlike source: one directly east and two to the west (see Figs. 3 and 4). Figure 3 shows a raw (unbinned, unsmoothed) total image of the nebula, and Figure 4 shows the hard (3.0-8.0 keV) emission smoothed by a 1 " FWHM Gaussian. Spatial fits with Sherpa to a twodimensional Gaussian indicate that the central source has a size consistent with a circular Gaussian of FWHM $=3.66$ pixels (1".8), while a point-spread function on the same region of the chip can be fitted with a Gaussian of 1.89 pixels (0".93) FWHM. This may be because the source is in fact resolved, but it may also be due to an ad hoc spatial model imposed on a region of complex morphology (a $20 \times 20$ pixel region surrounding the pointlike source was fitted with a two-dimensional constant, a circular Gaussian for the source itself, plus two elliptical Gaussians for the nebular emission surrounding the source).

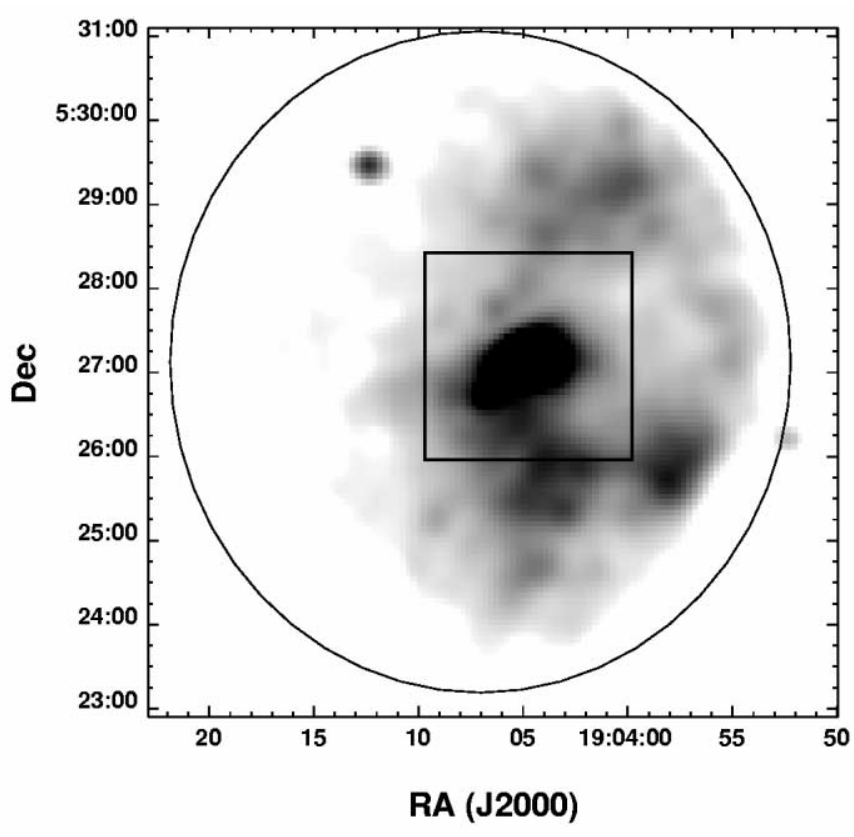

FIG. 2.-Broadband (0.5-8.0 keV) X-ray image of the remnant. The elliptical region shows the radio extent of the remnant. This $\mathrm{X}$-ray image has been binned by a factor of 4 and smoothed with a Gaussian with $5^{\prime \prime}$ FWHM. The image is scaled linearly from $5 \sigma$ to the maximum emission from the remnant shell. The box indicates the region of the PWN shown in Fig. 3.

\subsection{Central Nebula Spectrum}

The spectrum of the entire central nebula was extracted from an elliptical region surrounding the central pointlike source, as shown in Figure 3. Background was subtracted using an elliptical annulus (also indicated in Fig. 3) to remove thermal emission from the surrounding SNR shell. Approximately 3300 counts

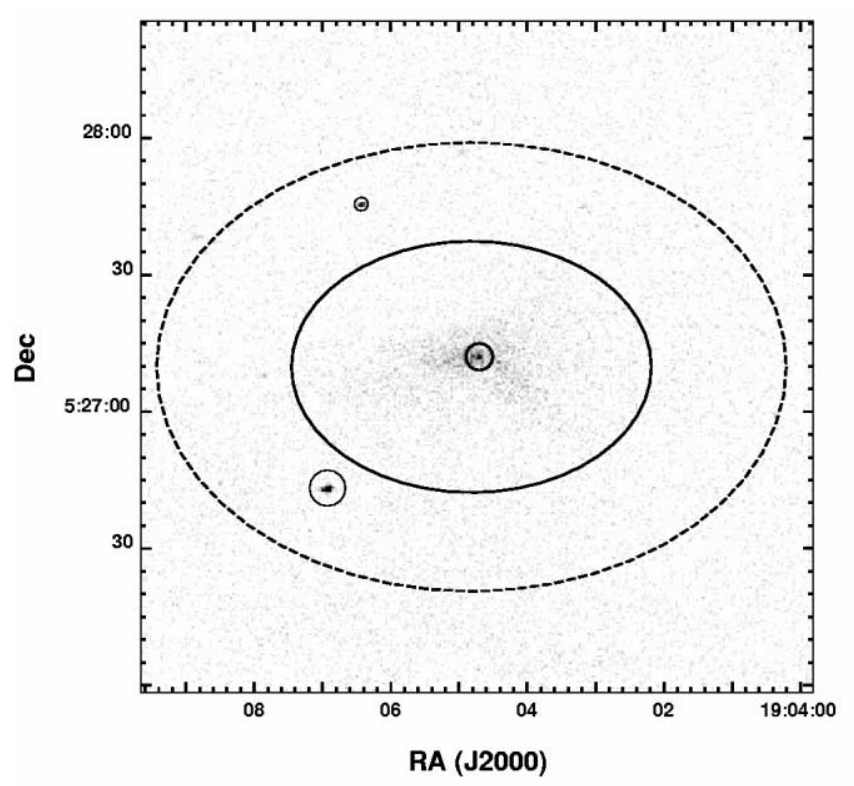

FIG. 3.-Raw imaging data of the central PWN from 0.3 to $8.0 \mathrm{keV}$, centered at $\alpha=19^{\mathrm{h}} 04^{\mathrm{m}} 04.7, \delta=05^{\circ} 27^{\prime} 11^{\prime \prime} .8$. This figure is plotted on a linear scale with its scale maximum defined by the pointlike source within the nebula. The regions used for spectral analysis are shown on the image as such: the inner 3 " circle defines the "pointlike" extraction region, while the solid ellipse surrounding it shows the PWN extraction region. The exterior dashed-line ellipse shows the outer boundary of the background used between the ellipses, excluding the two circled point sources. 


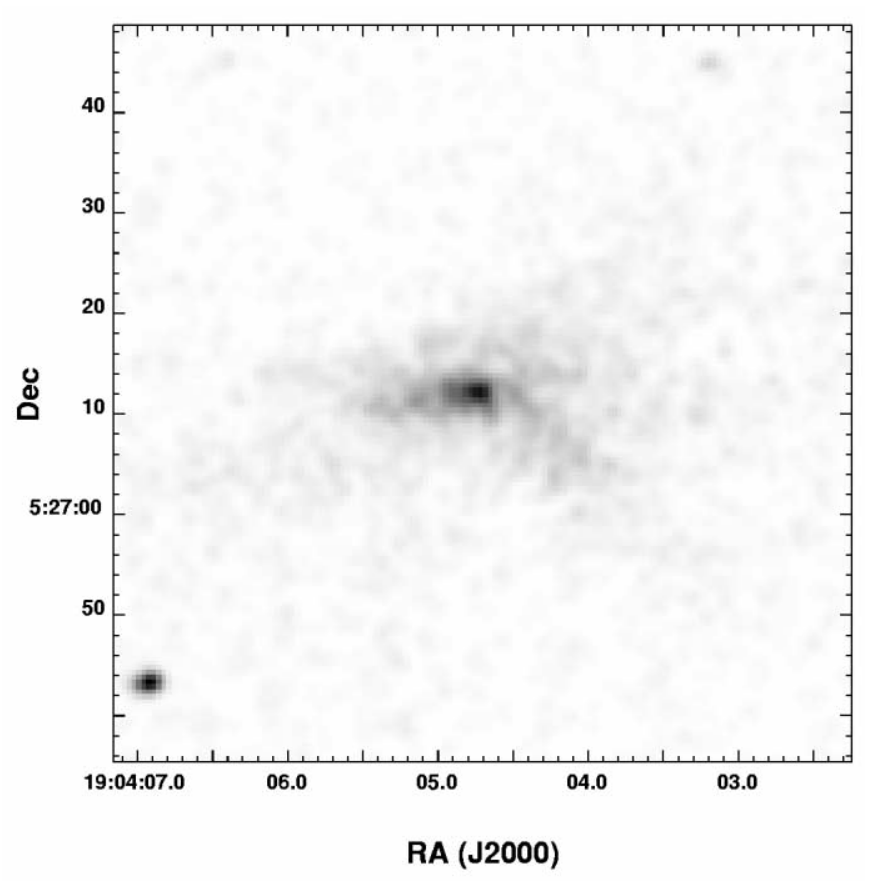

FIG. 4.- Hard-band (3.0-8.0 keV) image of the nebula smoothed by a 1 " FWHM Gaussian and scaled linearly with the maximum defined by the greatest number of counts at the center of the PWN. The extent of the PWN, particularly to the southwest, is most visible in this image.

were detected from the nebula and central source, after background subtraction. Spectra were grouped into bins with a minimum of 25 counts. The spectrum is satisfactorily characterized by a single absorbed power law using the absorption model of Morrison \& McCammon (1983; the XSPEC model "wabs"). The best-fit model from the $0.5-7.5 \mathrm{keV}$ spectrum yielded a column density of $N_{\mathrm{H}}=(5.3 \pm 0.9) \times 10^{22} \mathrm{~cm}^{-2}$ and a nebular photon index of $\Gamma_{\mathrm{PWN}+\text { point }}=1.5 \pm 0.3$ (at the $90 \%$ confidence level) for a $\chi_{v}^{2}=1.13$ (i.e., $\chi^{2}=51$ for 45 degrees of freedom). This column density is consistent with that obtained by Harrus \& Slane (1999) at the $1 \sigma$ level. This power-law photon index is significantly smaller (flatter) than that obtained in a fit with $A S C A$ data by Harrus \& Slane (1999), which may be the result of the greater ease of spatial differentiation between the shell and the nebula allowed by Chandra. In any case, the central nebula's spectrum is clearly distinct from that of the thermal SNR shell since there are no emission-line features in the former.

The spectrum of the pointlike source alone (the extraction region is also shown in Fig. 3 ) yields $\Gamma_{\text {point }}=1.2 \pm 0.25$; with the pointlike source excluded from the nebula extraction region, the remaining nebular emission has $\Gamma_{\mathrm{PWN}}=1.5 \pm 0.15$. The absorption column for the preceding two fits was fixed to the nebular value of $5.3 \times 10^{22} \mathrm{~cm}^{-2}$. The difference between the spectral indices of the pointlike source and those of the surrounding nebular emission is not statistically significant, although one might expect the slopes to differ (e.g., Gotthelf \& Olbert 2002; Gotthelf 2003). The 2-10 keV fluxes for the pointlike source and the nebula without the source are $S_{\text {point }}=$ $3.65 \times 10^{-13} \mathrm{ergs} \mathrm{cm}^{-2} \mathrm{~s}^{-1}$ and $S_{\text {nebula }}=1.39 \times 10^{-12}$ ergs $\mathrm{cm}^{-2} \mathrm{~s}^{-1}$, respectively.

\section{DISCUSSION}

The images obtained by the Chandra X-Ray Observatory clearly show a hard spectrum, elongated nebula within the Xray shell, and there is diffuse emission in the same region in the radio map. The most natural interpretation for such a non- thermal nebula is synchrotron emission from a shocked pulsar wind confined by the external pressure of the surrounding medium. The presence of such a nonthermal PWN distinguishes the associated neutron star from other objects such as anomalous X-ray pulsars and soft $\gamma$-ray repeaters (Marsden \& White 2001; Gaensler et al. 2001) and suggests that the nebula is powered by the rotational spin-down power of a young pulsar, confirming the interpretation of the nonthermal spectral component given by Harrus \& Slane (1999). With these new data, 3C 396 has joined the growing list of SNRs toward which the Chandra X-Ray Observatory has confirmed a previously unresolved neutron star/PWN candidate.

In $\S 2$, we discussed power-law fits to the nebula and the pointlike source. While several models provide statistically acceptable fits to the spectrum, we can eliminate some on physical grounds. A thermal bremsstrahlung model would require an extremely high temperature $(\sim 20 \mathrm{keV})$. A single blackbody characterizes the source (with $k T=1.2 \mathrm{keV} ; \chi_{\nu}^{2} \sim 1.25$ ), but this temperature is far higher than one would expect from a neutron star ( 0.1 keV; see Page 1995); such low-temperature thermal emission would be almost completely absorbed at such a high column density. Therefore, we dismiss the possibility of thermal emission from the nebula and the neutron star and conclude that the emission from the PWN is nonthermal synchrotron emission characterized by an absorbed power law.

Possenti et al. (2002) derive an empirical relationship between PWN luminosity and pulsar spin-down power: $\log L_{\mathrm{X}}=1.34 \log \dot{E}-15.34$, where $L_{\mathrm{X}}$ is in the $2-10 \mathrm{keV}$ band. We obtain an unabsorbed flux in this range of $S_{\mathrm{X}}=$ $1.62 \times 10^{-12}$ ergs $\mathrm{cm}^{-2} \mathrm{~s}^{-1}$ and thus an X-ray luminosity $L_{\mathrm{X}}$ in this range of $1.24 \times 10^{34} d_{8}^{2}$ ergs $\mathrm{s}^{-1}$, implying that $\dot{E}=7.2 \times 10^{36} d_{8}^{2}$ ergs s$^{-1}$, well in the range of other known pulsars and PWNe (Becker \& Pavlov 2003). This estimate for $\dot{E}$, after correcting for the reduction in distance, is approximately $50 \%$ lower than the estimate made by Harrus \& Slane (1999). The difficulty in separating the shell emission from the nebular emission with the low spatial resolution of ASCA may be responsible for this discrepancy. The inferred spin-down power and distance to this source suggest that this object may be the origin of the $\gamma$-ray emission powering the unidentified EGRET source 2EGS J1903+ 0529 (Yadigaroglu \& Romani 1997) based on its value of $\dot{E}_{33} / d_{\mathrm{kpc}}^{2} \simeq 110$ (where $\dot{E}_{33}$ is $\dot{E}$ in units of $10^{33} \mathrm{ergs} \mathrm{s}^{-1}$ and $d_{\mathrm{kpc}}$ is the distance to the source in units of kiloparsecs). Values of $\dot{E}_{33} / d_{\mathrm{kpc}}^{2}<0.5$ indicate a low probability of detection in the $\gamma$-ray, and bright $\gamma$-ray pulsars typically have such values well over 0.5 (Thompson 2000).

A similar calculation for $\dot{E}$ is often performed using the radio luminosity of the PWN, but this is infeasible here. In the case of 3C 396, the PWN was not observed in radio intensity but rather only in the polarization (Patnaik et al. 1990). In addition, a flatter spectral index was reported for the central region by Anderson \& Rudnick (1993). This flat central spectral index was measured using an unconventional sliding box method that is sensitive only to regions containing significant radio structure (Anderson \& Rudnick 1993). However, this method may depend on the visibility-plane coverage of the data, as appears to be the case for 3C 397 (Dyer \& Reynolds 1999b). There is also a possibility of thermal contamination from an extension of the flatter spectrum "plume" (Patnaik et al. 1990) that can be seen against the remnant itself (Anderson \& Rudnick 1993). Thus, the radio brightness of the PWN may be significantly less than the SNR in the same region, especially if the SNR has a smooth structure with low polarization, and the PWN is highly structured and polarized. At any rate, simply extrapo- 
lating the X-ray power-law model to radio frequencies suggests a radio flux density of about $1 / 25$ of the measured corresponding radio flux density, also supporting the picture of the PWN being only a small component of the radio central emission.

Assuming Harrus \& Slane's estimate for the remnant age of $t \sim 7000 \mathrm{yr}$ (i.e., $t_{7}=t / 7 \mathrm{kyr}$ ), a spin-down power of $10^{37}$ $\operatorname{ergs~s}{ }^{-1}\left(\dot{E}\right.$ in units of $1 \times 10^{37} \mathrm{ergs} \mathrm{s}^{-1}$, i.e., $\dot{E}_{37}=\dot{E} / 10^{37}$ ergs $\mathrm{s}^{-1}$ ), a standard moment of inertia of $I=10^{45} \mathrm{~g} \mathrm{~cm}^{2}$ (i.e., a standard neutron star radius of $10 \mathrm{~km}$ and a mass of $\left.1.4 M_{\odot}\right)$, and a braking index of $3(n=3)$, we estimate a pulsar period of $P=0.095[2 /(n-1)]^{1 / 2}\left(\dot{E}_{37} t_{7}\right)^{-1 / 2}$ s. Correspondingly, we estimate a period derivative of $\dot{P}=$ $P /[(n-1) t]=6.8 \times 10^{-13}[2 /(n-1)]^{1 / 2} \dot{E}_{37}^{-1 / 2} t_{7}^{-3 / 2} \mathrm{~s} \mathrm{~s}^{-1}$, which allows us to nominally estimate a dipolar magnetic field strength of $B=3.2 \times 10^{19}(P \dot{P})^{1 / 2}=4.4 \times 10^{13} \dot{E}_{37}^{-1 / 2} t_{7.1}^{-1} \mathrm{G}$.

A search for spectral variations (e.g., to search for spectral steepening as a function of radius from the pointlike source due to synchrotron aging or expansion losses) in the PWN is statistically infeasible. Figure 4 shows the hard-band image of the nebula that suggests the small-scale structure variations within the nebula. The elongations to the east and southwest may be polar outflows from the pulsar itself that are less confined than those observed in the Crab. The overall morphology is indicative of outflows reminiscent of Crab-like pulsars.

Given the age estimate of $7100 \mathrm{yr}$ and our previous distance estimate, and assuming that the center of the radio shell (which lies to the east of the nebula, away from the brighter edge) is the blast center of the remnant, we find an offset $\theta$ of $35^{\prime \prime}$ for the pointlike source, giving a velocity of $v \sim 190 d_{8} t_{7}^{-1} \mathrm{~km} \mathrm{~s}^{-1}$. Given the gradient in brightness from west to east, this is likely an upper limit since a density gradient might shift the blast center toward the nebula. This low velocity may explain why no clear bow shock morphology is seen. Thus, we are unable to constrain the location of the SNR blast center with these data.

\section{CONCLUSION}

We have analyzed a 100 ks observation of the Galactic supernova remnant 3C 396 and concluded from spectral and spatial analysis that the nonthermal emission detected by Harrus \& Slane (1999) is due to the presence of a PWN powered by a yet undetected pulsar. These data also support the notion that higher resolution and sensitivity instruments in the X-ray will continue to improve on the number of confirmed compact object-SNR associations and will allow a better determination of compact object birthrates. Future observations in the radio and X-rays may detect pulses from the pointlike source at the center of this nebula.

Support for this work was provided by the National Aeronautics and Space Administration through Chandra Award 2500834 issued by the Chandra X-ray Center, which is operated by the Smithsonian Astrophysical Observatory for and on behalf of the National Aeronautics Space Administration under contract NAS8-39073.

\section{REFERENCES}

Anderson, M. C., \& Rudnick, L. 1993, ApJ, 408, 514

Becker, R. H., \& Helfand, D. J. 1987, AJ, 94, 1629

Becker, W., \& Pavlov, G. G. 2003, in The Century of Space Science, ed. J. Bleeker, J. Geiss, \& M. Huber (Dordrecht: Kluwer), in press (astro-ph/ 0208356)

Biggs, J. D., \& Lyne, A. G. 1996, MNRAS, 282, 691

Caswell, J. L., Haynes, R. F., Milne, D. K., \& Wellington, K. J. 1982, MNRAS, 200, 1143

Caswell, J. L., Murray, J. D., Roger, R. S., Cole, D. J., \& Cooke, D. J. 1975, A\&A, 45, 239

Dyer, K. K., \& Reynolds, S. P. 1999a, BAAS, 31, 973 1999b, ApJ, 526, 365

Gaensler, B. M., Slane, P. O., Gotthelf, E. V., \& Vasisht, G. 2001, ApJ, 559, 963

Gorham, P. W., Ray, P. S., Anderson, S. B., Kulkarni, S. R., \& Prince, T. A. 1996, ApJ, 458, 257
Gotthelf, E. V. 2003, ArXiv Astrophys. e-print (3155)

Gotthelf, E. V., \& Olbert, C. M. 2002, in Proc. 270th WE-Heraeus Seminar on Neutron Stars, Pulsars, and Supernova Remnants, ed. W. H. Becker, H. Lesch, \& J. Trümper (Garching: MPE), 159

Harrus, I. M., \& Slane, P. O. 1999, ApJ, 516, 811

Marsden, D., \& White, N. E. 2001, ApJ, 551, L155

Morrison, R., \& McCammon, D. 1983, ApJ, 270, 119

Page, D. 1995, ApJ, 442, 273

Patnaik, A. R., Hunt, G. C., Salter, C. J., Shaver, P. A., \& Velusamy, T. 1990, A\&A, 232, 467

Possenti, A., Cerutti, R., Colpi, M., \& Mereghetti, S. 2002, A\&A, 387, 993

Thompson, D. J. 2000, Adv. Space Res., 25, 659

Townsley, L. K., Broos, P. S., Garmire, G. P., \& Nousek, J. A. 2000, ApJ, 534, L139

Yadigaroglu, I., \& Romani, R. W. 1997, ApJ, 476, 347 